\title{
KANNAN-TYPE FIXED POINT THEOREM IN CONE PENTAGONAL METRIC SPACES
}

\author{
Abba Auwalu ${ }^{1}$, Evren Hınçal ${ }^{2}$ \\ ${ }^{1,2}$ Department of Mathematics \\ Near East University \\ Nicosia-TRNC, Mersin 10, TURKEY
}

\begin{abstract}
In this paper, we prove Kannan - type fixed point theorem for two self mappings in non-normal cone pentagonal metric spaces. Our results extend and improve the recent results announced by many authors.
\end{abstract}

AMS Subject Classification: $47 \mathrm{H} 10,54 \mathrm{H} 25$

Key Words: cone pentagonal metric spaces, common fixed point, contraction mapping principle, weakly compatible maps

\section{Introduction}

A mapping $T: X \rightarrow X$ on a metric space $(X, d)$ is called Kannan contraction if there exists $\lambda \in[0,1 / 2)$ such that

$$
d(T x, T y) \leq \lambda[d(x, T x)+d(y, T y)], \text { for all } x, y \in X .
$$

Kannan [10] proved that if $X$ is complete, then every Kannan contraction has a fixed point.

The study of existence and uniqueness of fixed point of a mapping and common fixed points of two or more mappings has become a subject of great interest. Many authors proved the Kannan contraction principle in various generalized metric spaces (e.g., see $[2,7,8,11]$ ).

Received: March 9, 2016

Published: May 27, 2016

${ }^{\S}$ Correspondence author (c) 2016 Academic Publications, Ltd.

url: www.acadpubl.eu 
Long-Guang and Xian [7] introduced the concept of a cone metric space and proved some fixed point theorems for contractive type conditions in cone metric spaces. Later on many authors have (for e.g., [1, 3, 5, 6, 12]) proved some fixed point theorems for different contractive types conditions in cone metric spaces.

Recently, Garg and Agarwal [6] introduced the notion of cone pentagonal metric space and proved Banach contraction mapping principle in a normal cone pentagonal metric space setting.

Motivated and inspired by the results of $[6,11]$, it is our purpose in this paper to continue the study of common fixed points for a two self mappings in non-normal cone pentagonal metric space setting. Our results extend and improve the results of $[2,8,11]$, and many others.

\section{Preliminaries}

The following definitions and Lemmas, introduced in $[1,2,3,6,7]$, are needed in the sequel.

Definition 2.1. Let $E$ be a real Banach space and $P$ subset of $E$. $P$ is called a cone if and only if:

(1) $P$ is closed, nonempty, and $P \neq\{0\}$;

(2) $a, b \in \mathbb{R}, \quad a, b \geq 0$ and $x, y \in P \Longrightarrow a x+b y \in P$;

(3) $x \in P$ and $-x \in P \Longrightarrow x=0$.

Given a cone $P \subseteq E$, we defined a partial ordering $\leq$ with respect to $P$ by $x \leq y$ if and only if $y-x \in P$. We shall write $x<y$ to indicate that $x \leq y$ but $x \neq y$, while $x \ll y$ will stand for $y-x \in \operatorname{int}(P)$, where $\operatorname{int}(P)$ denotes the interior of $P$.

In this paper, we always suppose that $E$ is a real Banach space and $P$ is a cone in $E$ with $\operatorname{int}(P) \neq \emptyset$ and $\leq$ is a partial ordering with respect to $P$.

Definition 2.2. Let $X$ be a nonempty set. Suppose the mapping $\rho$ : $X \times X \rightarrow E$ satisfies:

(1) $0<\rho(x, y)$ for all $x, y \in X$ and $\rho(x, y)=0$ if and only if $x=y$;

(2) $\rho(x, y)=\rho(y, x)$ for all $x, y \in X$;

(3) $\rho(x, y) \leq \rho(x, z)+\rho(z, y)$ for all $x, y, z \in X$.

Then $\rho$ is called a cone metric on $X$, and $(X, \rho)$ is called a cone metric space. 
The concept of a cone metric space is more general than that of a metric space, because each metric space is a cone metric space where $E=\mathbb{R}$ and $P=[0, \infty)$ (e.g., see [7]).

Definition 2.3. Let $X$ be a nonempty set. Suppose the mapping $\rho$ : $X \times X \rightarrow E$ satisfies:

(1) $0<\rho(x, y)$ for all $x, y \in X$ and $\rho(x, y)=0$ if and only if $x=y$;

(2) $\rho(x, y)=\rho(y, x)$ for all $x, y \in X$;

(3) $\rho(x, y) \leq \rho(x, w)+\rho(w, z)+\rho(z, y)$ for all $x, y, z \in X$ and for all distinct points $w, z \in X-\{x, y\}$ [Rectangular property].

Then $\rho$ is called a cone rectangular metric on $X$, and $(X, \rho)$ is called a cone rectangular metric space.

Remark 2.4. Every cone metric space is cone rectangular metric space. The converse is not necessarily true (e.g., see [3]).

Definition 2.5. Let $X$ be a nonempty set. Suppose the mapping $d$ : $X \times X \rightarrow E$ satisfies:

(1) $0<d(x, y)$ for all $x, y \in X$ and $d(x, y)=0$ if and only if $x=y$;

(2) $d(x, y)=d(y, x)$ for $x, y \in X$;

(3) $d(x, y) \leq d(x, z)+d(z, w)+d(w, u)+d(u, y)$ for all $x, y, z, w, u \in X$ and for all distinct points $z, w, u, \in X-\{x, y\}$ [Pentagonal property].

Then $d$ is called a cone pentagonal metric on $X$, and $(X, d)$ is called a cone pentagonal metric space.

Remark 2.6. Every cone rectangular metric space and so cone metric space is cone pentagonal metric space. The converse is not necessarily true (e.g., see [6]).

Let $(X, d)$ be a cone pentagonal metric space. Let $\left\{x_{n}\right\}$ be a sequence in $(X, d)$ and $x \in X$. If for every $c \in E$ with $0 \ll c$ there exist $n_{0} \in \mathbb{N}$ and that for all $n>n_{0}, d\left(x_{n}, x\right) \ll c$, then $\left\{x_{n}\right\}$ is said to be convergent and $\left\{x_{n}\right\}$ converges to $x$, and $x$ is the limit of $\left\{x_{n}\right\}$. We denote this by $\lim _{n \rightarrow \infty} x_{n}=x$ or $x_{n} \rightarrow x$ as $n \rightarrow \infty$. If for every $c \in E$, with $0 \ll c$ there exist $n_{0} \in \mathbb{N}$ such that for all $n, m>n_{0}, d\left(x_{n}, x_{m}\right) \ll c$, then $\left\{x_{n}\right\}$ is called Cauchy sequence in $(X, d)$. If every Cauchy sequence is convergent in $(X, d)$, then $(X, d)$ is called a complete cone pentagonal metric space. 
Let $T$ and $S$ be self maps of a nonempty set $X$. If $w=T x=S x$ for some $x \in X$, then $x$ is called a coincidence point of $T$ and $S$ and $w$ is called a point of coincidence of $T$ and $S$. Also, $T$ and $S$ are said to be weakly compatible if they commute at their coincidence points, that is, $T x=S x$ implies that $T S x=S T x$.

Lemma 2.7. Let $T$ and $S$ be weakly compatible self mappings of nonempty set $X$. If $T$ and $S$ have a unique point of coincidence $w=T x=S x$, then $w$ is the unique common fixed point of $T$ and $S$.

Lemma 2.8. Let $(X, d)$ be a cone metric space with cone $P$ not necessary to be normal. Then for $a, c, u, v, w \in E$, we have

(1) If $a \leq h a$ and $h \in[0,1)$, then $a=0$.

(2) If $0 \leq u \ll c$ for each $0 \ll c$, then $u=0$.

(3) If $u \leq v$ and $v \ll w$, then $u \ll w$.

(4) If $c \in \operatorname{int}(P)$ and $a_{n} \rightarrow 0$, then $\exists n_{0} \in \mathbb{N}: \forall n>n_{0}, a_{n} \ll c$.

Lemma 2.9. Let $(X, d)$ be a complete cone pentagonal metric space. Let $\left\{x_{n}\right\}$ be a Cauchy sequence in $X$ and suppose that there is natural number $N$ such that:

1. $x_{n}=x_{m}$ for all $n, m>N$;

2. $x_{n}, x$ are distinct points in $X$ for all $n>N$;

3. $x_{n}, y$ are distinct points in $X$ for all $n>N$;

4. $x_{n} \rightarrow x$ and $x_{n} \rightarrow y$ as $n \rightarrow \infty$.

Then $x=y$.

\section{Main Results}

In this section, we prove Kannan - type contraction principle in cone pentagonal metric spaces for two self mappings. We give an example to illustrate the result.

Theorem 3.1. Let $(X, d)$ be a cone pentagonal metric space. Suppose the mappings $f, g: X \rightarrow X$ satisfy the contractive condition:

$$
d(f x, f y) \leq \lambda(d(g x, f x)+d(g y, f y))
$$


for all $x, y \in X$, where $\lambda \in[0,1 / 2)$. Suppose that $f(X) \subseteq g(X)$ and $g(X)$ or $f(X)$ is a complete subspace of $X$, then the mappings $f$ and $g$ have a unique point of coincidence in $X$. Moreover, if $f$ and $g$ are weakly compatible then $f$ and $g$ have a unique common fixed point in $X$.

Proof. Let $x_{0}$ be an arbitrary point in $X$. Since $f(X) \subseteq g(X)$, we can choose $x_{1} \in X$ such that $f x_{0}=g x_{1}$. Continuing this process, having chosen $x_{n}$ in $X$, we obtain $x_{n+1}$ in $X$ such that

$$
f x_{n}=g x_{n+1} \text { for all } n=0,1,2, \cdots .
$$

Now, we define a sequence $\left\{y_{n}\right\}$ in $X$ such that $y_{n}=f x_{n}=g x_{n+1}$ for all $n=$ $0,1,2, \cdots$. If $y_{k}=y_{k+1}$ for some $k \in \mathbb{N}$, then $y_{k}=f x_{k+1}=g x_{k+1}$. That is, $f$ and $g$ have a point of coincidence $y_{k}$ in $X$. We assume that $y_{n} \neq y_{n+1}$, for all $n \in \mathbb{N}$. Then, from (3.1), we have

$$
\begin{aligned}
d\left(y_{n}, y_{n+1}\right) & =d\left(f x_{n}, f x_{n+1}\right) \\
& \leq \lambda\left(d\left(g x_{n}, f x_{n}\right)+d\left(g x_{n+1}, f x_{n+1}\right)\right) \\
& =\lambda\left(d\left(y_{n-1}, y_{n}\right)+d\left(y_{n}, y_{n+1}\right)\right) .
\end{aligned}
$$

So that,

$$
\begin{aligned}
d\left(y_{n}, y_{n+1}\right) & \leq \frac{\lambda}{1-\lambda} d\left(y_{n-1}, y_{n}\right) \\
& \leq r d\left(y_{n-1}, y_{n}\right), \text { where } r=\frac{\lambda}{1-\lambda} \in[0,1) \\
& \leq r^{2} d\left(y_{n-2}, y_{n-1}\right) \\
& \vdots \\
& \leq r^{n}\left(d\left(y_{0}, y_{1}\right)\right), \quad \forall n \geq 1 .
\end{aligned}
$$

Also from (3.1) and (3.2), we obtain

$$
\begin{aligned}
d\left(y_{n}, y_{n+2}\right) & =d\left(f x_{n}, f x_{n+2}\right) \\
& \leq \lambda\left(d\left(g x_{n}, f x_{n}\right)+d\left(g x_{n+2}, f x_{n+2}\right)\right) \\
& \leq \lambda\left(d\left(y_{n-1}, y_{n}\right)+d\left(y_{n+1}, y_{n+2}\right)\right) \\
& \leq \lambda\left(r^{n-1} d\left(y_{0}, y_{1}\right)+r^{n+1} d\left(y_{0}, y_{1}\right)\right) \\
& \leq \lambda r^{n-1}\left(1+r^{2}\right) d\left(y_{0}, y_{1}\right) .
\end{aligned}
$$

That is,

$$
d\left(y_{n}, y_{n+2}\right) \leq \alpha r^{n-1} d\left(y_{0}, y_{1}\right), \quad \forall n \geq 1
$$


where $\alpha=\lambda\left(1+r^{2}\right)>0$.

For the sequence $\left\{y_{n}\right\}$, we consider $d\left(y_{n}, y_{n+p}\right)$ in two cases as follows:

If $p$ is odd say $p=2 m+1$, where $m \geq 1$, then by pentagonal property and (3.2), we have

$$
\begin{aligned}
d\left(y_{n}, y_{n+2 m+1}\right) \leq & d\left(y_{n}, y_{n+1}\right)+d\left(y_{n+1}, y_{n+2}\right)+d\left(y_{n+2}, y_{n+3}\right)+d\left(y_{n+3}, y_{n+2 m+1}\right) \\
\leq & d\left(y_{n}, y_{n+1}\right)+d\left(y_{n+1}, y_{n+2}\right)+d\left(y_{n+2}, y_{n+3}\right)+\cdots \\
& \quad+d\left(y_{n+2 m-1}, y_{n+2 m}\right)+d\left(y_{n+2 m}, y_{n+2 m+1}\right) \\
\leq & r^{n} d\left(y_{0}, y_{1}\right)+r^{n+1} d\left(y_{0}, y_{1}\right)+r^{n+2} d\left(y_{0}, y_{1}\right)+\cdots \\
& \quad+r^{n+2 m-1} d\left(y_{0}, y_{1}\right)+r^{n+2 m} d\left(y_{0}, y_{1}\right) \\
\leq & \frac{r^{n}}{1-r} d\left(y_{0}, y_{1}\right), \quad \forall n \geq 1 .
\end{aligned}
$$

If $p$ is even say $p=2 m$, where $m \geq 2$, then by pentagonal property, (3.2) and (3.3), we have

$$
\begin{aligned}
d\left(y_{n}, y_{n+2 m}\right) \leq & d\left(y_{n}, y_{n+2}\right)+d\left(y_{n+2}, y_{n+3}\right)+d\left(y_{n+3}, y_{n+4}\right)+d\left(y_{n+4}, y_{n+2 m}\right) \\
\leq & d\left(y_{n}, y_{n+2}\right)+d\left(y_{n+2}, y_{n+3}\right)+d\left(y_{n+3}, y_{n+4}\right)+\cdots \\
& \quad+d\left(y_{n+2 m-2}, y_{n+2 m-1}\right)+d\left(y_{n+2 m-1}, y_{n+2 m}\right) \\
\leq & \alpha r^{n-1} d\left(y_{0}, y_{1}\right)+r^{n+2} d\left(y_{0}, y_{1}\right)+r^{n+3} d\left(y_{0}, y_{1}\right)+\cdots \\
& \quad+r^{n+2 m-2} d\left(y_{0}, y_{1}\right)+r^{n+2 m-1} d\left(y_{0}, y_{1}\right) \\
\leq & \alpha r^{n-1} d\left(y_{0}, y_{1}\right)+\frac{r^{n}}{1-r} d\left(y_{0}, y_{1}\right) .
\end{aligned}
$$

Since $r \in[0,1)$, we get, as $n \rightarrow \infty, \frac{r^{\mathrm{n}}}{1-r} \rightarrow 0$ and $\alpha r^{n-1} \rightarrow 0$. Hence, for every $c \in E$ with $c \gg 0, \exists n_{0} \in \mathbb{N}$ such that

$$
d\left(y_{n}, y_{n+p}\right) \ll c, \text { for all } n \geq n_{0} .
$$

Therefore, $\left\{y_{n}\right\}$ is a Cauchy sequence in $(X, d)$. Since $g(X)$ is a complete subspace of $X$, there exists a points $u, v \in g(X)$ such that $\lim _{n \rightarrow \infty} y_{n}=v=g u$.

Now, we show that $g u=f u$. Given $c \gg 0$, we choose a natural numbers $M_{1}, M_{2}, M_{3}$ such that $d\left(v, y_{n}\right) \ll \frac{c(1-\lambda)}{3}, \forall n \geq M_{1}, d\left(y_{n}, y_{n+1}\right) \ll \frac{c(1-\lambda)}{3}, \forall n \geq$ $M_{2}$ and $d\left(y_{n+1}, y_{n+2}\right) \ll \frac{c(1-\lambda)}{3(1+\lambda)}, \quad \forall n \geq M_{3}$. Since $x_{n} \neq x_{m}$ for $n \neq m$, by pentagonal property, we have that

$$
\begin{aligned}
d(g u, f u) & \leq d\left(g u, y_{n}\right)+d\left(y_{n}, y_{n+1}\right)+d\left(y_{n+1}, y_{n+2}\right)+d\left(y_{n+2}, f u\right) \\
& \leq d\left(v, y_{n}\right)+d\left(y_{n}, y_{n+1}\right)+d\left(y_{n+1}, y_{n+2}\right)+d\left(f x_{n+2}, f u\right)
\end{aligned}
$$




$$
\begin{aligned}
\leq & d\left(v, y_{n}\right)+d\left(y_{n}, y_{n+1}\right)+d\left(y_{n+1}, y_{n+2}\right) \\
& +\lambda\left(d(g u, f u)+d\left(g x_{n+2}, f x_{n+2}\right)\right) \\
\leq & d\left(v, y_{n}\right)+d\left(y_{n}, y_{n+1}\right)+d\left(y_{n+1}, y_{n+2}\right) \\
& +\lambda\left(d(g u, f u)+d\left(y_{n+1}, y_{n+2}\right)\right) \\
d(g u, f u) \leq & \frac{1}{1-\lambda}\left(d\left(v, y_{n}\right)+d\left(y_{n}, y_{n+1}\right)+(1+\lambda) d\left(y_{n+1}, y_{n+2}\right)\right) \\
\ll & \frac{c}{3}+\frac{c}{3}+\frac{c}{3}=c, \text { for all } n \geq M,
\end{aligned}
$$

where $M:=\max \left\{M_{1}, M_{2}, M_{3}\right\}$. Since $c$ is arbitrary, we have $d(g u, f u) \ll$ $\frac{c}{m}, \forall m \in \mathbb{N}$. Since $\frac{c}{m} \rightarrow 0$ as $m \rightarrow \infty$, we conclude $\frac{c}{m}-d(g u, f u) \rightarrow-d(g u, f u)$ as $m \rightarrow \infty$. Since $P$ is closed, $-d(g u, f u) \in P$. Hence $d(g u, f u) \in P \cap-P$. By definition of cone we get that $d(g u, f u)=0$, and so $g u=f u=v$. Hence, $v$ is a point of coincidence of $f$ and $g$. Similarly, if $f(X)$ is a complete subspace of $X$ the result holds.

Next, we show that $v$ is unique. For suppose $v^{\prime}$ be another point of coincidence of $f$ and $g$, that is $g u^{\prime}=f u^{\prime}=v^{\prime}$, for some $u^{\prime} \in X$, then

$$
d\left(v, v^{\prime}\right)=d\left(f u, f u^{\prime}\right) \leq \lambda\left(d(g u, f u)+d\left(g u^{\prime}, f u^{\prime}\right)\right) \leq \lambda\left(d(v, v)+d\left(v^{\prime}, v^{\prime}\right)\right) .
$$

Hence, $v=v^{\prime}$. Since $(f, g)$ is weakly compatible, by Lemma 2.7, $v$ is the unique common fixed point of $f$ and $g$. This completes the proof of the theorem.

To illustrate Theorem 3.1, we give the following example.

Example 3.2. Let $X=\{a, b, c, d, e\}, E=\mathbb{R}^{2}$ and $P=\{(x, y): x, y \geq 0\}$ is a cone in $E$. Define $\rho: X \times X \rightarrow E$ as follows:

$$
\begin{aligned}
\rho(x, x) & =0, \forall x \in X ; \\
\rho(a, b) & =\rho(b, a)=(4,16) ; \\
\rho(a, c) & =\rho(c, a)=\rho(c, d)=\rho(d, c)=\rho(b, c)=\rho(c, b) \\
& =\rho(b, d)=\rho(d, b)=\rho(a, d)=\rho(d, a)=(1,4) ; \\
\rho(a, e) & =\rho(e, a)=\rho(b, e)=\rho(e, b)=\rho(c, e) \\
& =\rho(e, c)=\rho(d, e)=\rho(e, d)=(5,20) .
\end{aligned}
$$

Then $(X, \rho)$ is a complete cone pentagonal metric space, but $(X, \rho)$ is not a complete cone rectangular metric space because it lacks the rectangular property:

$$
(4,16)=\rho(a, b)>\rho(a, c)+\rho(c, d)+\rho(d, b)
$$




$$
\begin{aligned}
& =(1,4)+(1,4)+(1,4) \\
& =(3,12), \text { as }(4,16)-(3,12)=(1,4) \in P .
\end{aligned}
$$

Define a mapping $f, g: X \rightarrow X$ as follows:

$$
\begin{aligned}
& f(x)= \begin{cases}d, & \text { if } x \neq e \\
b, & \text { if } x=e\end{cases} \\
& g(x)= \begin{cases}c, & \text { if } x=a \\
a, & \text { if } x=b ; \\
b, & \text { if } x=c \\
d, & \text { if } x=d ; \\
e, & \text { if } x=e\end{cases}
\end{aligned}
$$

Clearly $f(X) \subseteq g(X)$ and $g(X)$ is a complete subspace of $X$. Also $f$ and $g$ are weakly compatible mappings. Thus, the conditions of Theorem 3.1 holds for all $x, y \in X$, where $\lambda=\frac{1}{5}$ and $d \in X$ is the unique common fixed point of the mappings $f$ and $g$.

Corollary 3.3. (see [2]) Let $(X, d)$ be a complete cone pentagonal metric space and $P$ be a normal cone with normal constant $k$. Suppose the mapping $S: X \rightarrow X$ satisfies the contractive condition:

$$
d(S x, S y) \leq \lambda(d(x, S x)+d(y, S y)),
$$

for all $x, y \in X$, where $\lambda \in[0,1 / 2)$. Then

1. $S$ has a unique fixed point in $X$.

2. For any $x \in X$, the iterative sequence $\left\{S^{n} x\right\}$ converges to the fixed point.

Proof. Take $g=I$ and $P$ be a normal cone in Theorem 3.1. This completes the proof.

Corollary 3.4. (see [11]) Let $(X, d)$ be a cone rectangular metric space and $P$ be a normal cone with normal constant $k$. Suppose the mappings $S, g$ : $X \rightarrow X$ satisfies the contractive condition:

$$
d(S x, S y) \leq \lambda(d(g x, S x)+d(g y, S y)),
$$

for all $x, y \in X$, where $\lambda \in[0,1 / 2)$. Suppose that $S(X) \subseteq g(X)$ and $S(X)$ or $g(X)$ is a complete subspace of $X$, then the mappings $S$ and $g$ have a unique coincidence point in $X$. Moreover, if $S$ and $g$ are weakly compatible then $S$ and $g$ have a unique common fixed point in $X$. 
Proof. This follows from Remark 2.6 and Theorem 3.1, where $P$ is a normal cone.

Corollary 3.5. (see [8]) Let $(X, d)$ be a complete cone rectangular metric space and $P$ be a normal cone with normal constant $k$. Suppose the mapping $S: X \rightarrow X$ satisfies the contractive condition:

$$
d(S x, S y) \leq \lambda(d(x, S x)+d(y, S y))
$$

for all $x, y \in X$, where $\lambda \in[0,1 / 2)$. Then

1. $S$ has a unique fixed point in $X$.

2. For any $x \in X$, the iterative sequence $\left\{S^{n} x\right\}$ converges to the fixed point.

Proof. Take $g=I$ and $P$ be a normal cone in Theorem 3.1 and Remark 2.6. This completes the proof.

\section{Acknowledgments}

This research project was supported by the Center of Excellence, Near East University, Nicosia-TRNC, Mersin 10, Turkey.

\section{References}

[1] M. Abbas, G. Jungck, Common fixed point results for non commuting mappings without continuity in cone metric spaces, J. Math. Anal. Appl., 341, No. 1 (2008), 416-420, doi: 10.1016/j.jmaa.2007.09.070.

[2] A. Auwalu, Kannan's fixed point theorem in a cone pentagonal metric space, J. Math. Comp. Sci., 6, (2016), Article ID 2562, 12 pages.

[3] A. Azam, M. Arshad, I. Beg, Banach contraction principle on cone rectangular metric spaces, Appl. Anal. Discrete Math., 3, (2009), 236-241, doi: 10.2298/AADM0902236A.

[4] S. Banach, Sur les opérations dans les ensembles abstraits et leur application aux équations intégrales, Fundamenta Mathematicae, 3, (1922), 133-181.

[5] E. Hınçal, A. Auwalu, A Note on Banach Contraction Mapping principle in Cone Hexagonal Metric Space, British J. Math. Comput. Sci., 16, No. 1 (2016), 1-12, doi: 10.9734/BJMCS/2016/25172.

[6] M. Garg, S. Agarwal, Banach Contraction Principle on Cone Pentagonal Metric Space, J. Adv. Stud. Topol., 3, No. 1 (2012), 12-18.

[7] L.G. Huang, X. Zhang, Cone metric spaces and fixed point theorems of contractive mappings, J. Math. Anal. Appl., 332, No. 2 (2007), 1468-1476, doi: 10.1016/j.jmaa.2005.03.087. 
[8] M. Jleli, B. Samet, The Kannan's fixed point theorem in a cone rectangular metric space, J. Nonlinear Sci. Appl., 2, No. 3 (2009), 161-167.

[9] R. Kannan, Some results on fixed point, Bull. Calcutta Math. Soc., 60, (1968), 71-76.

[10] R. Kannan, Some results on fixed points II, Amer. Math. Monthly, 76, (1969), 405-408.

[11] M.P. Reddy, M. Rangamma, A Common fixed point theorem for Two Self Maps in a Cone rectangular metric space, Bull. Math. Stat. Res., 3, No. 1 (2015), 47-53.

[12] S. Rezapour, R. Hamlbarani, Some notes on paper cone metric spaces and fixed point theorems of contractive mappings, J. Math. Anal. and Appl., 347, No. 2 (2008), 719-724, doi: 10.1016/j.jmaa.2008.04.049. 